\title{
Laparoscopic gastric pouch and remnant resection: a novel approach to refractory anastomotic ulcers after Roux-en-Y Gastric Bypass: Case report
}

\author{
Daniel C Steinemann ${ }^{1,2}$, Marc Schiesser ${ }^{1}$, Pierre-Alain Clavien ${ }^{1}$ and Antonio Nocito ${ }^{1 *}$
}

\begin{abstract}
Background: Anastomotic or marginal ulcers occur in 0.6 to $16 \%$ of patients after laparoscopic Roux-en-Y-Gastric Bypass. Initial therapy aims at eliminating known risk factors including smoking, Helicobacter pylori infection, use of non-steroidal anti-inflammatory drugs and inhibition of gastric acid secretion. While this approach is successful in 68 to $88 \%$ of the cases, up to one third of patients need a subsequent surgical revision. However, marginal ulcers still recur in up to $10 \%$ of cases after revisional surgery, thus constituting a serious challenge for bariatric surgeons.

Case presentation: We herein report a case of an insidious marginal ulcer refractory to both medical therapy with high-dosed proton pump inhibitors and sucralfate as well as surgical therapy consisting of the lengthening of a short alimentary limb and later resection of the gastroenterostomy and construction of a new tension-free anastomosis. Only after gastrectomy by laparoscopic en-bloc resection of the gastrojejunostomy, the gastric pouch and resection of the gastric remnant with reconstruction by esophagojejunostomy the patient remained free of symptoms.
\end{abstract}

Conclusion: By laparoscopic resection of the entire gastric pouch and the gastric remnant the risk to leave a suboptimally vascularised or even ischemic pouch in situ was avoided. The esophagojejunostomy was then created in healthy, good vascularised tissue. In our case this novel approach was effective in the management of a refractory anastomotic ulcer and might represent a rescue option when simple revision of the gastrojejunostomy fails.

Keywords: Roux-en-Y-Gastric Bypass, bariatric surgery, anastomotic ulcer, marginal ulcer, obesity

\section{Background}

A specific complication after laparoscopic Roux-en-YGastric-Bypass (LRYGB) is a marginal or anastomotic ulcer (AU) occurring at the gastrojejunal anastomosis. While AU can remain asymptomatic in $62-92 \%$ of the cases [1-4], they can frequently cause disabling pain or complications such as perforation and bleeding [5,6]. The incidence of AU varies from $0.6 \%$ to $16 \%$ in endoscopic studies [1-4].

\footnotetext{
* Correspondence: antonio.nocito@usz.ch

'Department of Visceral and Transplantation Surgery, University Hospital

Zurich, Raemistrasse 100, 8091 Zurich, Switzerland

Full list of author information is available at the end of the article
}

The etiology of AU is unclear. Two classes of risk factors have been suggested: operative and patient related factors. Although large gastric pouch, vertically oriented pouch [7], gastro-gastric fistula [8], local tissue ischemia due to anastomotic tension [9] or presence of foreign bodies in the ulcer ground (e.g. nonabsorbable sutures) [10] have been previously discussed, there is still a lack of high level of evidence demonstrating these factors to be significant. In contrast, better data exist for patient related factors, showing that smoking (odds ratio (OR) 30.6), use of nonsteroidal anti-inflammatory drugs (OR 11.5) and lack of proton pump inhibitor (PPI) prophylaxis (OR 3) represent significant risk factors [11].

\section{Biomed Central}


Therefore, ulcer therapy starts with elimination of patient related risk factors and inhibition of gastric acid secretion. While this approach is successful in 68 to $88 \%$, up to one third of the patients need a subsequent surgical revision $[8,12]$. Although revision surgery is successful in most cases, AU recur in up to $8 \%$ [8], thus leading to a distressing situation for both patients and bariatric surgeons.

We herein report a case of an insidious AU refractory medical and surgical therapy, which finally required an aggressive approach consisting of laparoscopic gastric pouch and gastric remnant resection.

\section{Case presentation}

In a 50 year male patient with a BMI of $45 \mathrm{~kg} / \mathrm{m}^{2}$ an antecolic, antegastric LRYGB with a $100 \mathrm{~cm}$ alimentary and a $60 \mathrm{~cm}$ biliary limb was performed. The gastrojejunostomy was constructed using a $25 \mathrm{~mm}$ circular stapler (EEA 2535, 3.5 mm Staples, Covidien ${ }^{\circledR}$ ). Simultaneously, a $6 \mathrm{~cm}$ silastic (Fobi) ring was placed around the gastrojejunostomy. A few weeks after discharge, the patient, who continued smoking after surgery, presented with strong epigastric pain, postprandial regurgitation and vomiting. He was unable to eat solid food and to attend work. Endoscopy revealed two AU at the gastrojejunostomy. Oral PPI therapy (esomeprazole, $80 \mathrm{mg} / \mathrm{die}$ ) was initiated and, since it was thought to be partly responsible for the symptoms, the silastic ring was removed. Intravenous high-dose PPI (esomeprazole, $240 \mathrm{mg} / \mathrm{die}$ ) led to healing of the AU. However, epigastric pain and regurgitation did not ameliorate. A $99 \mathrm{~m}$ Tc-mebrofenin scintigraphy revealed severe biliary reflux. Seven months after LRYGB the patient was referred to our department.

At the initial consultation the patient was taking up to $600 \mathrm{mg} /$ day esomeprazole and $200 \mathrm{mg} /$ day tilidin orally. As an AU could not be detected further diagnostic investigations were performed:

- Upper gastrointestinal (GI) contrast series revealing a small gastric pouch without signs of a gastric fistula and normal passage.

- Double balloon push enteroscopy demonstrating a short $(40 \mathrm{~cm})$ alimentary limb.

- High-resolution esophageal manometry revealing a normotensive propulsive peristalsis and a normotense lower esophageal sphincter.

- 24 h-impedance pH-metry - performed under antacid medication - showing no pathological acid or nonacid reflux.

- MRI in Sellink technique showing no obstruction of the small bowel.

Apart from the biliary reflux diagnosed by scintigraphy consistent with a very short Roux-limb by push-enteroscopy, no other reason for the epigastric pain was detected. We performed a laparoscopic lengthening of the Roux limb by additional $120 \mathrm{~cm}$ resulting in a new alimentary limb of $160 \mathrm{~cm}$. Oral PPI therapy $(80 \mathrm{mg} /$ day) was continued and sucralfate $(4 \mathrm{~g} / \mathrm{d})$ was added.

After a short period without pain and regurgitation, symptoms recurred two weeks after Roux limb lengthening. Despite PPI therapy endoscopy revealed a recurrent $\mathrm{AU}$ at the gastrojejunostomy (Figure 1). Therefore a laparoscopic resection of the gastrojejunostomy was performed followed by a construction of a new, tensionfree anastomosis using a $25 \mathrm{~mm}$ circular stapler (EEA 2535, 3.5 mm Staples, Covidien $\left.{ }^{\circledR}\right)$. PPI therapy and sucralfate were continued. Again the patient was discharged free of symptoms on postoperative day five.

One month later and one year after initial LRYGB surgery, the patient was again not free of epigastric pain. Gastroscopy showed again a large AU at the gastroenterostomy. Meanwhile, the patient was finally motivated enough to quit smoking and was enrolled in a stopsmoking program. Since gastrin level was not elevated (111 ng/l) an underlying gastrinoma could be excluded. Furthermore, Helicobacter pylori and hyperparathyroidism as additional potential causes for anastomotic ulcers were also ruled out. Nevertheless, epigastric pain and the AU persisted.

At this point an aggressive approach was decided consisting of a gastrectomy by laparoscopic en-bloc resection of the gastrojejunostomy and the gastric pouch with transsection $2 \mathrm{~cm}$ proximal to the angle of His and resection of the gastric remnant (Figure 2). The gastrointestinal continuity was re-established by the construction of an esophagojejunostomy using a $25 \mathrm{~mm}$ circular stapler (Figure 3). Two days after surgery an upper GI contrast series showed no leakage or stenosis at the level of the esophagojejunostomy. The patient was

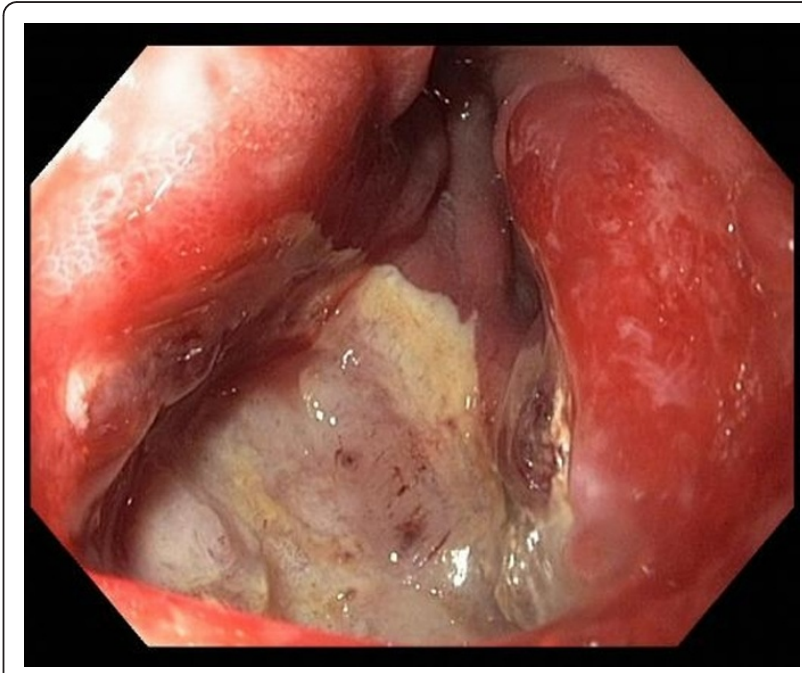

Figure 1 Recurrent anastomotic ulcer in the intestinal part of the gastrojejunostomy. 


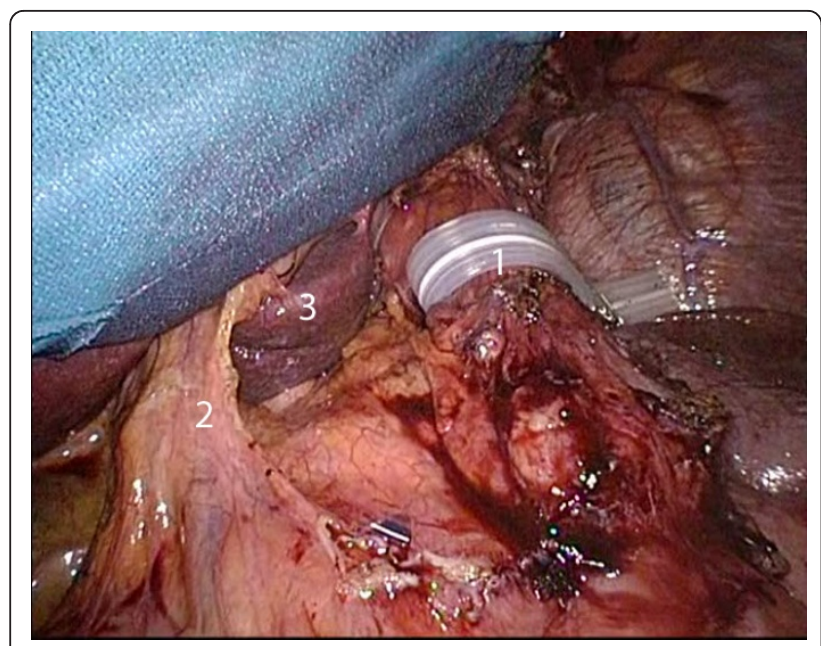

Figure 2 Situs after en-bloc resection of the gastric pouch and the gastrojejunostomy. ( 1 = esophagus, 2 = hepatoduodenal ligament, 3 = caudate lobe).

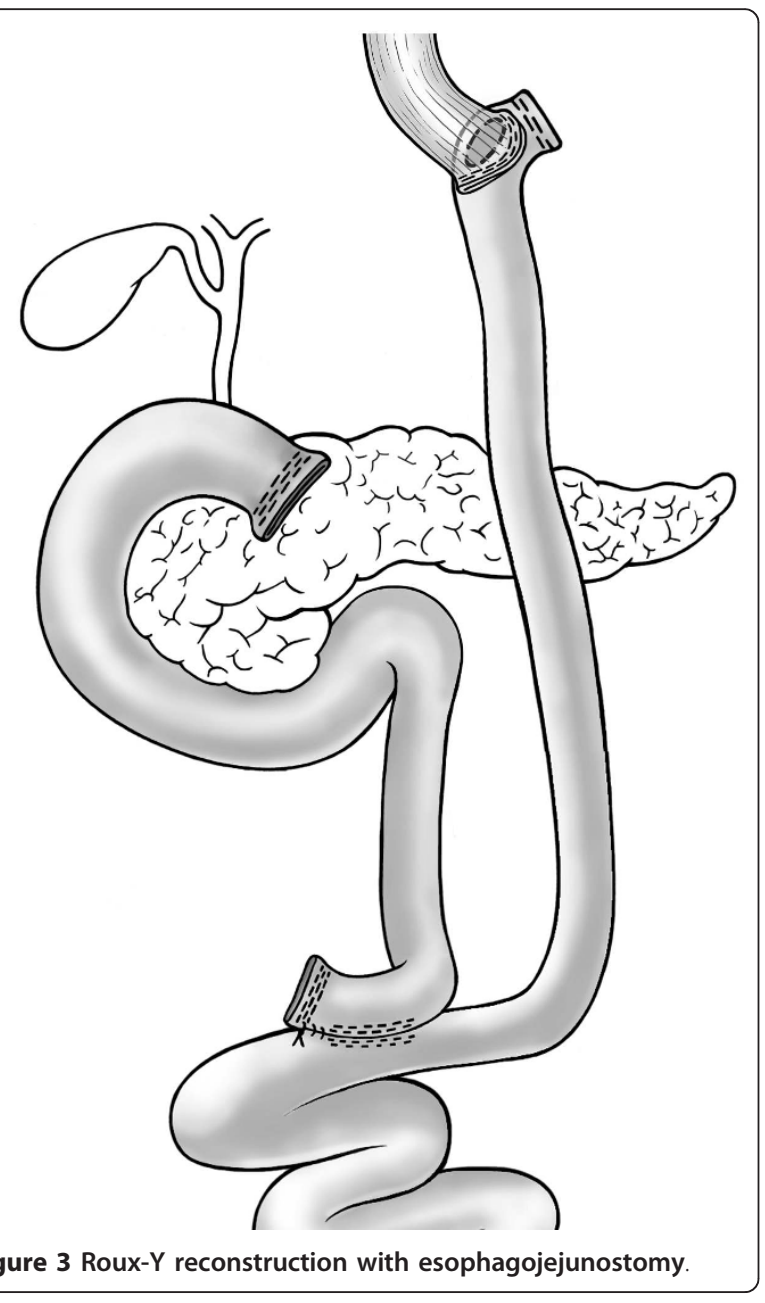

discharged on postoperative day 10. Six months later the patient was free of symptoms, he was able to start opioid weaning and had regained $6 \mathrm{~kg}$ of weight. Finally, endoscopy showed a regular esophagojejunostomy.

\section{Discussion and conclusion}

Our case of a persistently recurring AU is representative for the current shortcomings in understanding the pathogenesis and thus optimal treatment of AU. We describe a successful approach for the management of intractable AU.

After LRYGB up to 7\% of patients develop upper GI symptoms. Analysis and management of this condition is often challenging as $32 \%$ of symptomatic patients show a normal anatomy at endoscopy [13]. Conservative therapy has been reported to be successful in $68 \%-88 \%$ of the cases $[8,12]$. In our case, endoscopy showed no abnormalities apart from a short Roux limb at the time of referral. As a short Roux limb may facilitate biliary reflux causing postoperative pain and AU [14], we decided to lengthen the alimentary limb. Despite improvement of regurgitation symptoms, epigastric pain and AU recurred.

Approximately one third of AU recur after medical therapy. For these cases a redo of the gastrojejunostomy with a success rate of $87 \%$ has been advised [8]. Before we embarked on this strategy, we reevaluated whether putative factors leading to a recurrence were present. Endoscopy and GI contrast series excluded potential operative risk factors. Therefore a resection of the gastrojejunostomy with subsequent PPI therapy was performed. However, the ulcer recurred potentially due to the inability of the patient to quit smoking.

After revision surgery for AU, a recurrence rate of $8 \%$ has been described. In these cases, revision of the gastrojejunostomy combined with gastric remnant resection has been advocated reducing gastrin-producing- and parietal cells [8].

In contrast to the proposed simple revision of the anastomosis, we opted for a laparoscopic resection of the entire gastric pouch and the gastric remnant. By this means, the risk to leave a suboptimally vascularised or even ischemic pouch in situ was avoided since the resection was taken back to esophageal tissue. The circular esophagojejunostomy was then created in healthy, good vascularised tissue. Subsequently, the patient was free of symptoms and no recurrence was observed after a follow-up of 6 months. Hence, in our case this novel approach was effective in the management of a refractory AU and might represent a rescue option when simple revision of the gastrojejunostomy fails.

\section{Consent}

Written informed consent was obtained from the patient for publication of this case report and any 
accompanying images. A copy of the written consent is available for review by the Editor-in-Chief of this journal.

\section{Acknowledgements}

We thank Marco Bueter and Paul Schneider of the Department of Visceral and Transplantation Surgery, University Hospital Zurich, for the critical review of the manuscript. This study is not externally funded by government or charity foundations. No funding or assistance is received from commercial organizations.

Permissions

None of the material has been previously published.

\section{Author details}

'Department of Visceral and Transplantation Surgery, University Hospital Zurich, Raemistrasse 100, 8091 Zurich, Switzerland. '2Department of Surgery, Cantonal Hospital Bruderholz, 4104 Bruderholz, Switzerland

\section{Authors' contributions}

DCS drafted and finalized the manuscript, MS and PAC reviewed the manuscript, AN performed the surgery, monitored the drafting and critically reviewed the manuscript and has given final approve for publication. All authors read and approved the final manuscript.

\section{Competing interests}

The authors declare that they have no competing interests. No financial support has been received.

Received: 21 August 2011 Accepted: 2 December 2011 Published: 2 December 2011

\section{References}

1. Garrido AB Jr, Rossi M, Lima SE Jr, Brenner AS, Gomes CA Jr: Early marginal ulcer following Roux-en- $Y$ gastric bypass under proton pump inhibitor treatment: prospective multicentric study. Arq Gastroenterol 2010, 47(2):130-134

2. Csendes A, Burgos AM, Altuve J, Bonacic S: Incidence of marginal ulcer 1 month and 1 to 2 years after gastric bypass: a prospective consecutive endoscopic evaluation of 442 patients with morbid obesity. Obes Surg 2009, 19(2):135-138.

3. Vasquez JC, Wayne Overby D, Farrell TM: Fewer gastrojejunostomy strictures and marginal ulcers with absorbable suture. Surg Endosc 2009, 23(9):2011-2015.

4. D'Hondt MA, Pottel H, Devriendt D, Van Rooy F, Vansteenkiste F: Can a short course of prophylactic low-dose proton pump inhibitor therapy prevent stomal ulceration after laparoscopic Roux-en-Y gastric bypass? Obes Surg 2010, 20(5):595-599.

5. Wheeler AA, de la Torre RA, Fearing NM: Laparoscopic repair of perforated marginal ulcer following Roux-en-Y gastric bypass: a case series. J Laparoendosc Adv Surg Tech A 2011, 21(1):57-60.

6. Avgerinos DV, Llaguna $\mathrm{OH}$, Seigerman M, Lefkowitz AJ, Leitman IM: Incidence and risk factors for the development of anemia following gastric bypass surgery. World J Gastroenterol 2010, 16(15):1867-1870.

7. Sapala JA, Wood MH, Sapala MA, Flake TM Jr: Marginal ulcer after gastric bypass: a prospective 3-year study of 173 patients. Obes Surg 1998, 8(5):505-516

8. Patel RA, Brolin RE, Gandhi A: Revisional operations for marginal ulcer after Roux-en-Y gastric bypass. Surg Obes Relat Dis 2009, 5(3):317-322.

9. Nguyen NT, Hinojosa M, Fayad C, Varela E, Wilson SE: Use and outcomes of laparoscopic versus open gastric bypass at academic medical centers. J Am Coll Surg 2007, 205(2):248-255.

10. Sacks BC, Mattar SG, Qureshi FG, Eid GM, Collins JL, Barinas-Mitchell EJ, Schauer PR, Ramanathan RC: Incidence of marginal ulcers and the use of absorbable anastomotic sutures in laparoscopic Roux-en-Y gastric bypass. Surg Obes Relat Dis 2006, 2(1):11-16.

11. Wilson JA, Romagnuolo J, Byrne TK, Morgan K, Wilson FA: Predictors of endoscopic findings after Roux-en-Y gastric bypass. Am J Gastroenterol 2006, 101(10):2194-2199.
12. Carrodeguas L, Szomstein S, Soto F, Whipple O, Simpfendorfer C, Gonzalvo JP, Villares A, Zundel N, Rosenthal R: Management of gastrogastric fistulas after divided Roux-en-Y gastric bypass surgery for morbid obesity: analysis of 1,292 consecutive patients and review of literature. Surg Obes Relat Dis 2005, 1(5):467-474.

13. Lee JK, Van Dam J, Morton JM, Curet M, Banerjee S: Endoscopy is accurate, safe, and effective in the assessment and management of complications following gastric bypass surgery. Am J Gastroenterol 2009, 104(3):575-582, quiz 583.

14. Swartz DE, Mobley E, Felix EL: Bile reflux after Roux-en-Y gastric bypass: an unrecognized cause of postoperative pain. Surg Obes Relat Dis 2009, 5(1):27-30.

\section{Pre-publication history}

The pre-publication history for this paper can be accessed here: http://www.biomedcentral.com/1471-2482/11/33/prepub

doi:10.1186/1471-2482-11-33

Cite this article as: Steinemann et al:: Laparoscopic gastric pouch and remnant resection: a novel approach to refractory anastomotic ulcers after Roux-en-Y Gastric Bypass: Case report. BMC Surgery 2011 11:33.

\section{Submit your next manuscript to BioMed Central and take full advantage of:}

- Convenient online submission

- Thorough peer review

- No space constraints or color figure charges

- Immediate publication on acceptance

- Inclusion in PubMed, CAS, Scopus and Google Scholar

- Research which is freely available for redistribution 